\title{
Essentials for Great Teams: Trust, Diversity, Communication ... and Joy
}

\author{
Linda M. Roth, PhD, and Tsveti Markova, MD
}

In clinics and hospitals, in conference rooms and classrooms, in hallways and lunchrooms, we demonstrate daily our effectiveness or ineffectiveness as team members. We interact with patients, coworkers, and learners in ways that result in smooth, well-functioning, patient-centered processes, or we interact in ways that impede those processes. This "hidden curriculum" is powerful and teaches others about our competence in and commitment to teamwork. The recent focus on teams in patient care, education, and research calls for increasing awareness of our own roles in settings in which we have responsibilities that are mutual to or complementary with others'.

In the minds of many physicians - even those who have committed to a team approach to health care-there may still be a number of questions about how a team should function and what the obligations and behaviors of each team member should be. As Baker and colleagues ${ }^{1}$ noted in their review of teams in health care, the medical community frequently has focused on measuring outcomes, such as the time between arrival and admission or errors in procedures, rather than on "process measures"-the activities, strategies, responses, and behaviors of team members as they function in teams. Although these are more difficult to collect and quantify, a number of these processes have been demonstrated to contribute to effective teamwork in health care settings. Foundational processes for teamwork include a practice's development of and commitment to a strong sense

Submitted 2 December 2011; revised 2 December 2011; accepted 5 December 2011.

From the Department of Family Medicine and Public Health Sciences, Wayne State University School of Medicine, Detroit, MI.

Funding: none.

Conflict of interest: none declared.

Corresponding author: Linda M. Roth, PhD, 3939 Woodward, \#323, Detroit, MI 48201 (E-mail: lroth@med. wayne.edu). of trust, inclusion of diverse perspectives in clinical operations and decision making, and strategic use of a continuum of effective communication strategies. The synergy that results from developing and sustaining successful teams can result in a satisfying, even joyful, work environment.

\section{Trust}

What does it take to develop trust among clinic team members? Physicians may ask themselves, Do I trust my medical assistant to take an accurate social history and document it appropriately in the chart? Do I double check the nurse's recommendation for patient self-management? Do I allow the receptionist to lead a new check-out process? Am I less of a leader if I don't have all the answers about the patient flow within my office or don't start all improvement initiatives myself? At the individual level, the answers to these questions depend on how much we trust our team colleagues and are willing to be vulnerable to one another. ${ }^{2}$ Feeling safe being vulnerable to our colleagues requires that we understand each other's roles and allow each staff member to operate freely within his or her scope of practice ${ }^{3}$ while relying on frequent, meaningful dialogue across all members of the group who are, together, accountable for patient care. $^{4}$

\section{Diversity}

A patient's experience in any clinic is influenced by individuals who vary greatly in their training, roles, and experience. Including relevant staff members in decision making regarding quality improvement, practice change, and clinical operations has been shown to positively influence practice productivity and reduce staff turnover. ${ }^{5}$ Gallagher and colleagues ${ }^{6}$ capitalized on differing strengths and perspectives of clinic employees in 16 small primary care practices. They trained 2 coleaders (a physician and a nonphysician) in each practice to imple- 
ment improvements in depression care and found that such a structure allowed coleaders to optimize their complementary skills, relationships, and credibility in implementing and sustaining practice improvements. Similarly, in a study of 42 primary care practices in the United Kingdom, practices whose team members rated their team climate more highly than others were more likely to provide higher quality care for diabetes and elicit more positive patient evaluations of their practices. ${ }^{7}$ A positive team climate encourages people to speak up and share their ideas and in fact engenders team members to become leaders of those projects for which they have passion, knowledge, and skill. Diversity—not only of race, sex, and culture, but of training and experience-is embraced in a team-focused environment. This diversity of perspectives enhances group problem solving and creativity. ${ }^{2}$ Herein lies the opportunity to transition from good to great, from a focus on a group of employees who are expected to follow a powerful leader to an interdependent team who together lead a thriving and enduring organization. 8 , The concept of "distributed leadership"4 may seem foreign to physicians whose training and professional development focuses on individual accountability in making high-stakes, critical decisions regarding their patients. Whether in the operating room, hospital ward, or ambulatory clinic, physicians can consciously work to trust and empower their fellow health care team members, who together with physicians are striving toward the same outcome: the health and well-being of patients. An inclusive perspective can foster true collaboration, where all team members are empowered to participate in analyzing problems and generating solutions. ${ }^{4}$ Both "group" culture, based on norms and values associated with affiliation, teamwork, and participation, and "developmental" culture, based on risk-taking, innovation, and change, have been shown to affect more positively the quality of patient care than cultures based on hierarchy or efficiency and achievement. ${ }^{10}$

\section{Communication}

Throughout the work day, members of a clinic's team routinely make observations about how to improve elements of patient care. Although these ideas may be shared in momentary exchanges, they rarely result in changes in the process of care. This illustrates one of the most important barriers to effective improvement processes: the lack of dedicated time for exchange of ideas among those directly responsible for each clinic process.

Prioritizing regular time periods for all employees to contribute to practice discussions allows a team to determine positive changes in a practice and to sustain those changes over time. ${ }^{11}$ In addition to face-to-face encounters during practice and written or electronic notes and records, successful health care teams develop a variety of effective communication methods ranging from team huddles at the beginning of the day, to regularly scheduled team meetings, to practice retreats that include all who contribute to patient care. Across this continuum, effective teams dedicate themselves to facilitating real involvement of employees at all levels. Primary care teams especially have benefited from providing members the authority to manage work the way team members wish to and ensuring that everyone can participate and influence improvements. ${ }^{12} \mathrm{In}$ Craigie and Hobbs ${ }^{13}$ exploration of 2 community health practices that had been identified as exemplary, weekly team meetings were described as "safe places where employees could raise issues and receive respectful, collaborative, problem-solving responses."

Brown and colleagues ${ }^{14}$ interviewed a wide variety of employees in primary health care teams in Canada to determine activities that foster sustained health care teams. Formal meetings, professional development programs, and retreats were seen as essential both to strengthen relationships among members and to assure that effective communication was taking place. Interviewees particularly valued the social interactions made possible by retreats, and many noted with regret that limited funding and time had begun to reduce or eliminate the possibility of such activities. Social activities interspersed throughout the clinic day or for special occasions also were seen as contributing to bonding team members together, as did openly sharing life events-happy ones such as weddings as well as times of illness and loss.

\section{Joy}

Trust, diversity, and communication are fundamental principles of effective teamwork in health care. On this foundation, clinic teams can develop an exceptional environment that fosters enhanced quality of care for patients in tandem with en- 
hanced quality of life for the team members who deliver that care. Positive relationships among employees can contribute to a culture, or "spirit," that benefits employees, their process of clinical care, and their patients. ${ }^{13}$ Given that a recent large-scale study of primary care teams in the Veterans Administration system found that aggregate measures of team members' job satisfaction were significantly and positively related to quality of the process of patient care as well as quality of outcomes of patient care ${ }^{15}$ we have more reason than ever to focus on the people with whom we work as well as the on the work we do as individuals. A new construct, "joy in work," recently emerged in a study of successful teams within chronic care collaboratives. ${ }^{16}$ This may be the harbinger of better lives for both caregivers and patients; "joy in work" comprises positive experiences and outcomes among the combined team of physicians, nonphysician team members, and their patients.

\section{Conclusion}

We know that trust, diversity of perspectives, and effective communication are fundamental to establishing and sustaining a team culture throughout the clinical environment. The importance of doing so is clear, and the responsibility for doing so lies with each of us. We need to make time to attend to not only what we do, but how we work together. Working as a true team, we can improve the processes and outcomes of patient care as well as increase each person's joy in work-the satisfaction that comes with a job well done, in concert with our colleagues and our patients.

\section{References}

1. Baker DP, Salas E, Battles J, Barch P. The role of teamwork in the professional education of physicians: current status and assessment recommendations. Jt Comm J Qual Patient Saf 2005;31:185-202.

2. Lanham HJ, McDaniel RR Jr, Crabtree BF, et al. How improving practice relationships among clinicians and nonclinicians can improve quality in primary care. Jt Comm J Qual Patient Saf 2009;35:457-66.
3. Brown J, Lewis L, Ellis K, Stewart M, Freeman TR, Kasperski MJ. Conflict on interprofessional primary health care teams-can it be resolved? J Interprof Care 2011;25:4-10.

4. Johnson PA, Bookman A, Bailyn L, Harrington M, Orton P. Innovation in ambulatory care: a collaborative approach to redesigning the health care workplace. Acad Med 2011;86:211-6.

5. Hung DY, Rundall TG, Cohen DJ, Tallia AF, Crabtree BF. Productivity and turnover in PCPs: the role of staff participation in decision-making. Med Care 2006;44:946-51.

6. Gallagher K, Nutting PA, Nease DE Jr, et al. It takes two: using coleaders to champion improvements in small primary care practices. J Am Board Fam Med 2010;23:632-9.

7. Bower P, Campbell S, Bojke C, Sibbald B. Team structure, team climate and the quality of care in primary care: an observational study. Qual Saf Health Care 2003;12:273-9.

8. Collins JC. Good to great: why some companies make the leap-and others don't. New York, NY: Harper Business; 2001.

9. Katzenbach J, Smith D. The discipline of teams. Harvard Bus Rev. 2005;83:162-71.

10. Howard M, Brazil K, Akhtar-Danesh N, Agarwal G. Self-reported teamwork in family health team practices in Ontario: organizational and cultural predictors of team climate. Can Fam Physician 2011;57: e185-91.

11. Stroebel CK, McDaniel RR, Crabtree BF, Miller WL, Nutting PA, Stange KC. How complexity science can inform a reflective process for improvement in primary care practices. Jt Comm J Qual Patient Saf 2005;31:438-46.

12. Lukas CV, Mohr DC, Meterko M. Team effectiveness and organizational context in the implementation of a clinical innovation. Qual Manag Health Care 2009;18:25-39.

13. Craigie FC Jr, Hobbs RF 3rd. Exploring the organizational culture of exemplary community health center practices. Fam Med 200;36:733-8.

14. Brown JB, Lewis L, Ellis K, et al. Sustaining primary health care teams: what is needed? J Interprof Care 2010;24:463-5.

15. Mohr DC, Young GJ, Meterko M, Stolzmann KL, White B. Job satisfaction of primary care team members and quality of care. Am J Med Qual 2011;26: $18-25$.

16. Johnson JK, Woods DM, Stevens DP, et al. Joy and challenges in improving chronic illness care: capturing daily experiences of academic primary care teams. J Gen Intern Med 2010;25(Suppl 4):S581-5. 